\title{
Cohomology of the adjoint of Hopf algebras
}

\author{
J. Scott CARTER ${ }^{a}$, Alissa S. CRANS ${ }^{b}$, Mohamed ELHAMDADI $^{c}$, and Masahico SAITO ${ }^{c}$ \\ ${ }^{a}$ University of South Alabama, Mobile, AL 36688, USA \\ Email: carter@jaguar1.usouthal.edu \\ ${ }^{b}$ Loyola Marymount Unversity, Los Angeles, CA 90045, USA \\ E-mail: acrans@lmu.edu \\ ${ }^{c}$ University of South Florida, Tampa, FL 33620, USA \\ E-mails: emohamed@math.usf.edu and saito@math.usf.edu
}

\begin{abstract}
A cohomology theory of the adjoint of Hopf algebras, via deformations, is presented by means of diagrammatic techniques. Explicit calculations are provided in the cases of group algebras, function algebras on groups, and the bosonization of the super line. As applications, solutions to the YBE are given and quandle cocycles are constructed from groupoid cocycles.
\end{abstract}

\section{Introduction}

Algebraic deformation theory [10] can be used to define 2-dimensional cohomology in a wide variety of contexts. This theory has also been understood diagrammatically $[7,16,17]$ via PROPs, for example. In this paper, we use diagrammatic techniques to define a cohomological deformation of the adjoint $\operatorname{map} \operatorname{ad}(x \otimes y)=\sum S\left(y_{(1)}\right) x y_{(2)}$ in an arbitrary Hopf algebra. We have concentrated on the diagrammatic versions here because diagrammatics have led to topological invariants $[6,13,19]$, diagrammatic methodology is prevalent in understanding particle interactions and scattering in the physics literature, and most importantly kinesthetic intuition can be used to prove algebraic identities.

The starting point for this calculation is a pair of identities that the adjoint map satisfies and that are sufficient to construct Woronowicz's solution [22] $R=(1 \otimes \mathrm{ad})(\tau \otimes 1)(1 \otimes \Delta)$ to the Yang-Baxter equation (YBE): $(R \otimes 1)(1 \otimes R)(R \otimes 1)=(1 \otimes R)(R \otimes 1)(1 \otimes R)$. We use deformation theory to define an extension 2-cocycle. Then we show that the resulting 2-coboundary map, when composed with the Hochschild 1-coboundary map is trivial. A 3-coboundary is defined via the "movie move" technology. Applications of this cohomology theory include constructing new solutions to the YBE by deformations and constructing quandle cocycles from groupoid cocycles that arise from this theory.

The paper is organized as follows. Section 2 reviews the definition of Hopf algebras, defines the adjoint map, and illustrates Woronowicz's solution to the YBE. Section 3 contains the deformation theory. Section 4 defines the chain groups and differentials in general. Example calculations in the case of a group algebra, the function algebra on a group, and a calculation of the 1- and 2-dimensional cohomology of the bosonization of the superline are presented in Section 5. Interestingly, the group algebra and the function algebra on a group are cohomologically different. Moreover, the conditions that result when a function on the group algebra satisfies the cocycle condition coincide with the definition of groupoid cohomology. This relationship is given in Section 6, along with a construction of quandle 3-cocycles from groupoid 3-cocycles. In Section 7 , we use the deformation cocycles to construct solutions to the Yang-Baxter equation. 


\section{Preliminaries}

We begin by recalling the operations and axioms in Hopf algebras, and their diagrammatic conventions depicted in Figures 1 and 2.

A coalgebra is a vector space $C$ over a field $k$ together with a comultiplication $\Delta: C \rightarrow C \otimes C$ that is bilinear and coassociative: $(\Delta \otimes 1) \Delta=(1 \otimes \Delta) \Delta$. A coalgebra is cocommutative if the comultiplication satisfies $\tau \Delta=\Delta$, where $\tau: C \otimes C \rightarrow C \otimes C$ is the transposition $\tau(x \otimes y)=y \otimes x$. A coalgebra with counit is a coalgebra with a linear map called the counit $\epsilon: C \rightarrow k$ such that $(\epsilon \otimes 1) \Delta=1=(1 \otimes \epsilon) \Delta$ via $k \otimes C \cong C$. A bialgebra is an algebra $A$ over a field $k$ together with a linear map called the unit $\eta: k \rightarrow A$, satisfying $\eta(a)=a \mathbf{1}$ where $\mathbf{1} \in A$ is the multiplicative identity and with an associative multiplication $\mu: A \otimes A \rightarrow A$ that is also a coalgebra such that the comultiplication $\Delta$ is an algebra homomorphism. A Hopf algebra is a bialgebra $C$ together with a map called the antipode $S: C \rightarrow C$ such that $\mu(S \otimes 1) \Delta=\eta \epsilon=\mu(1 \otimes S) \Delta$, where $\epsilon$ is the counit.

In diagrams, the compositions of maps are depicted from bottom to top. Thus a multiplication $\mu$ is represented by a trivalent vertex with two bottom edges representing $A \otimes A$ and one top edge representing $A$. Other maps in the definition are depicted in Fig. 1 and axioms are depicted in Fig. 2.

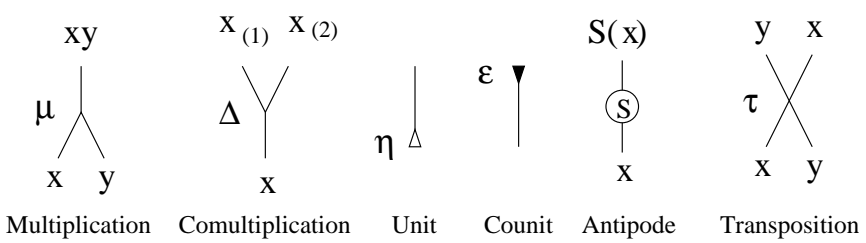

Figure 1: Operations in Hopf algebras

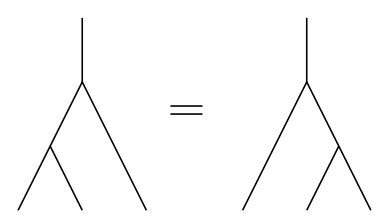

Associativity

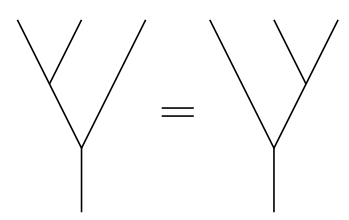

Coassociativity

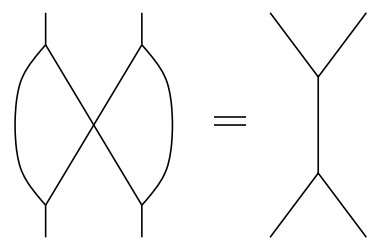

Compatibility

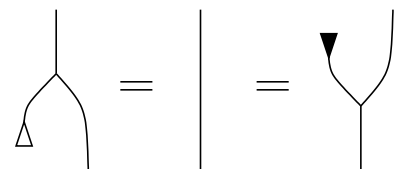

Unit

Counit<smiles>CC#CC(C)CC</smiles>

Unit is a coalgebra hom

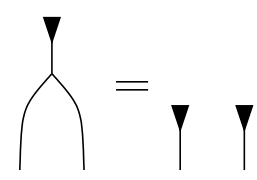

Counit is an algebra hom

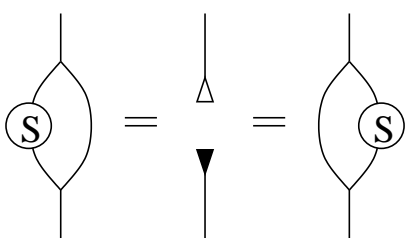

Antipode condition

Figure 2: Axioms of Hopf algebras

Let $H$ be a Hopf algebra. The adjoint map $\operatorname{Ad}_{y}: H \rightarrow H$ for any $y \in H$ is defined by $\operatorname{Ad}_{y}(x)=S\left(y_{(1)}\right) x y_{(2)}$, where we use the common notation $\Delta(x)=x_{(1)} \otimes x_{(2)}$ and $\mu(x \otimes y)=x y$. Its diagram is depicted in Fig. $3(\mathrm{~A})$. Notice the analogy with group conjugation: in a group ring $H=k G$ over a field $k$, where $\Delta(y)=y \otimes y$ and $S(y)=y^{-1}$, we have $\operatorname{Ad}_{y}(x)=y^{-1} x y$. For the adjoint map ad $: H \otimes H \rightarrow H$ we have $\operatorname{ad}(x \otimes y)=\operatorname{Ad}_{y}(x)$.

Definition 1. Let $H$ be a Hopf algebra and ad be the adjoint map. Then the linear map 


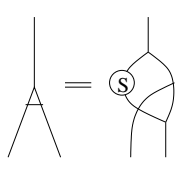

(A)

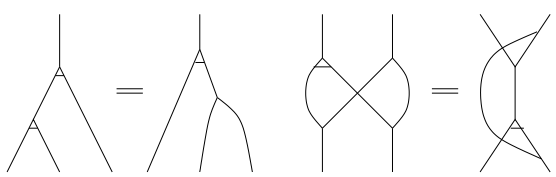

(B)

Figure 3: Conditions for the YBE for Hopf algebras

$R_{\text {ad }}: H \otimes H \rightarrow H \otimes H$ defined by $R_{\text {ad }}=(1 \otimes \mathrm{ad})(\tau \otimes 1)(1 \otimes \Delta)$ is said to be the $R$-matrix induced from ad.

Lemma 1. The R-matrix induced from ad satisfies the YBE.

Proof. In Fig. 4, it is indicated that the YBE follows from two properties of the adjoint map:

$$
\begin{aligned}
& \operatorname{ad}(\operatorname{ad} \otimes 1)=\operatorname{ad}(1 \otimes \mu) \\
& (\operatorname{ad} \otimes \mu)(1 \otimes \tau \otimes 1)(\Delta \otimes \Delta)=(1 \otimes \mu)(\tau \otimes 1)(1 \otimes \Delta)(1 \otimes \operatorname{ad})(\tau \otimes 1)(1 \otimes \Delta)
\end{aligned}
$$

It is known that these properties are satisfied, and proofs are found in $[11,22]$. Here we include diagrammatic proofs for reader's convenience in Fig. 5 and Fig. 6, respectively.

Definition 2. We call the equalities in equations (2.1) and (2.2) the adjoint conditions.

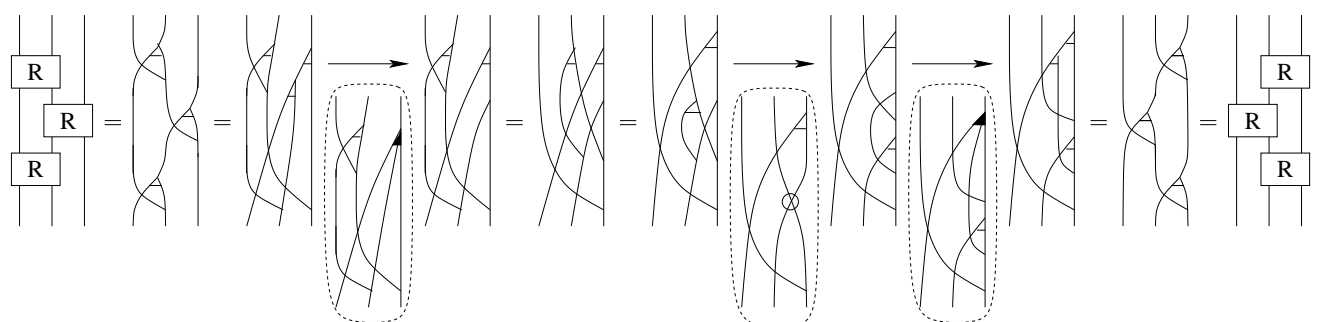

Figure 4: YBE by the adjoint map

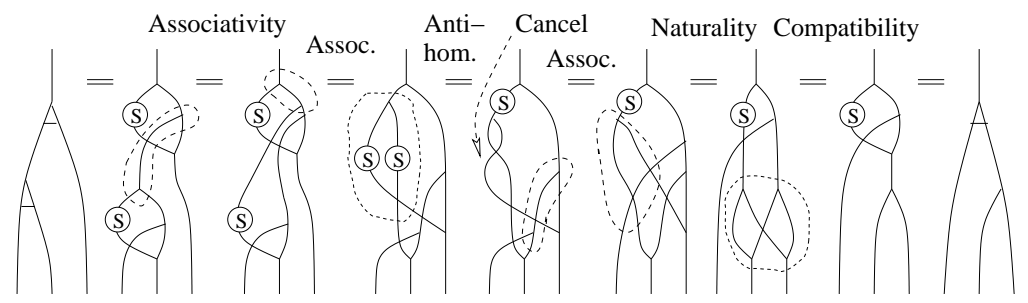

Figure 5: Adjoint condition (1)

Remark 1. The equality (2.1) is equivalent to the fact that the adjoint map defines an algebra action of $H$ on itself (see [14]). Specifically, $(a \triangleleft b) \triangleleft c=a \triangleleft(b c)$ for any $a, b, c \in H$, where $\triangleleft$ denotes the right action defined by the adjoint: $a \triangleleft b=\operatorname{ad}(a \otimes b)$. The equality (2.2) can be similarly rewritten as

$$
a_{(1)} \triangleleft b_{(1)} \otimes a_{(2)} b_{(2)}=\left(a \triangleleft b_{(2)}\right)_{(1)} \otimes b_{(1)}\left(a \triangleleft b_{(2)}\right)_{(2)}
$$

Remark 2. It was pointed out to us by Sommerhaeuser that the induced $R$-matrix $R_{\text {ad }}$ is invertible with inverse $R_{\text {ad }}^{-1}(b \otimes a)=b_{(3)} a S^{-1}\left(b_{(2)}\right) \otimes b_{(1)}$. 


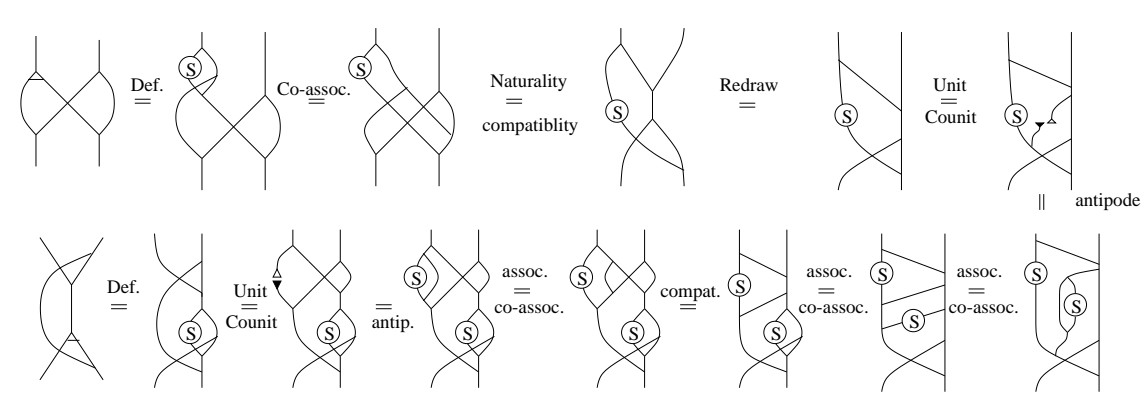

Figure 6: Adjoint condition (2)

\section{Deformations of the adjoint map}

We follow the exposition in [16] for deformation of bialgebras to propose a similar deformation theory for the adjoint map. In light of Lemma 1, we deform the two equalities (2.1) and (2.2). Let $H$ be a Hopf algebra and ad its adjoint map.

Definition 3. A deformation of $(H$, ad $)$ is a pair $\left(H_{t}, \operatorname{ad}_{t}\right)$ where $H_{t}$ is a $k[[t]]$-Hopf algebra given by $H_{t}=H \otimes k[[t]]$ with all Hopf algebra structures inherited by extending those on $H_{t}$ with the identity on the $k[[t]]$ factor (the trivial deformation as a Hopf algebra), with a deformations of ad given by $\operatorname{ad}_{t}=\mathrm{ad}+\operatorname{tad}_{1}+\cdots+t^{n} \operatorname{ad}_{n}+\cdots: H_{t} \otimes H_{t} \rightarrow H_{t}$, where $\operatorname{ad}_{i}: H \otimes H \rightarrow H$, $i=1,2, \cdots$, are maps.

Suppose $\overline{a d}=\operatorname{ad}+\cdots+t^{n} \operatorname{ad}_{n}$ satisfies the adjoint conditions (equalities (2.1) and (2.2)) mod $t^{n+1}$, and suppose that there exist $\operatorname{ad}_{n+1}: H \otimes H \rightarrow H$ such that ad $+t^{n+1} \operatorname{ad}_{n+1}$ satisfies the adjoint conditions mod $t^{n+2}$. Define $\xi_{1} \in \operatorname{Hom}\left(H^{\otimes 3}, H\right)$ and $\xi_{2} \in \operatorname{Hom}\left(H^{\otimes 2}, H^{\otimes 2}\right)$ by

$$
\begin{aligned}
& \overline{\operatorname{ad}}(\overline{\operatorname{ad}} \otimes 1)-\overline{\operatorname{ad}}(1 \otimes \mu)=t^{n+1} \xi_{1} \quad \bmod t^{n+2} \\
& (\overline{\operatorname{ad}} \otimes \mu)(1 \otimes \tau \otimes 1)(\Delta \otimes \Delta)-(1 \otimes \mu)(\tau \otimes 1)(1 \otimes \Delta)(1 \otimes \overline{\mathrm{ad}})(\tau \otimes 1)(1 \otimes \Delta)=t^{n+1} \xi_{2} \quad \bmod t^{n+2}
\end{aligned}
$$

For the first adjoint condition (2.1) of ad $+t^{n+1} \operatorname{ad}_{n+1} \bmod t^{n+2}$ we obtain

$$
\left(\left(\overline{\mathrm{ad}}+t^{n+1} \mathrm{ad}_{n+1}\right) \otimes 1\right)-\left(\overline{\mathrm{ad}}+t^{n+1} \mathrm{ad}_{n+1}\right)(1 \otimes \mu)=0 \bmod t^{n+2}
$$

which is equivalent by degree calculations to

$$
\operatorname{ad}\left(\operatorname{ad}_{n+1} \otimes 1\right)+\operatorname{ad}_{n+1}(\operatorname{ad} \otimes 1)-\operatorname{ad}_{n+1}(1 \otimes \mu)=\xi_{1}
$$

For the second adjoint condition (2.2) of ad $+t^{n+1} \operatorname{ad}_{n+1} \bmod t^{n+2}$ we obtain

$$
\begin{aligned}
& \left(\left(\overline{\mathrm{ad}}+t^{n+1} \mathrm{ad}_{n+1}\right) \otimes \mu\right)(1 \otimes \tau \otimes 1)(\Delta \otimes \Delta) \\
& \quad-(1 \otimes \mu)(\tau \otimes 1)(1 \otimes \Delta)\left(1 \otimes\left(\overline{\mathrm{ad}}+t^{n+1} \mathrm{ad}_{n+1}\right)\right)(\tau \otimes 1)(1 \otimes \Delta)=0 \quad \bmod t^{n+2}
\end{aligned}
$$

which is equivalent by degree calculations to

$$
\left(\operatorname{ad}_{n+1} \otimes \mu\right)(1 \otimes \tau \otimes 1)(\Delta \otimes \Delta)-(1 \otimes \mu)(\tau \otimes 1)(1 \otimes \Delta)\left(1 \otimes \operatorname{ad}_{n+1}\right)(\tau \otimes 1)(1 \otimes \Delta)=\xi_{2}
$$

In summary we proved the following.

Lemma 2. The map ad $+t^{n+1} \operatorname{ad}_{n+1}$ satisfies the adjoint conditions mod $t^{n+2}$ if and only if

$$
\begin{aligned}
& \operatorname{ad}\left(\operatorname{ad}_{n+1} \otimes 1\right)+\operatorname{ad}_{n+1}(\operatorname{ad} \otimes 1)-\operatorname{ad}_{n+1}(1 \otimes \mu)=\xi_{1} \\
& \left(\operatorname{ad}_{n+1} \otimes \mu\right)(1 \otimes \tau \otimes 1)(\Delta \otimes \Delta)-(1 \otimes \mu)(\tau \otimes 1)(1 \otimes \Delta)\left(1 \otimes \operatorname{ad}_{n+1}\right)(\tau \otimes 1)(1 \otimes \Delta)=\xi_{2}
\end{aligned}
$$

We interpret this lemma as $\left(\xi_{1}, \xi_{2}\right)$ being the primary obstructions to formal deformation of the adjoint map. In the next section we will define coboundary operators, and show that $\left(\xi_{1}, \xi_{2}\right)=(0,0)$ gives that $\operatorname{ad}_{n+1}$ is a 2 -cocycle, and that $\left(\xi_{1}, \xi_{2}\right)$ satisfies the 3 -cocycle condition, just as in the case of deformations and Hochshild cohomology for bialgebras. 


\section{Differentials and cohomology}

\subsection{Chain groups}

We define chain groups, for positive integers $n, n>1$, and $i=1, \ldots, n$ by

$$
C_{\mathrm{ad}}^{n, i}(H ; H)=\operatorname{Hom}\left(H^{\otimes(n+1-i)}, H^{\otimes i}\right), \quad C_{\mathrm{ad}}^{n}(H ; H)=\oplus_{i>0, i \leq n+1-i} C_{a d}^{n, i}(H ; H)
$$

Specifically, chain groups in low dimensions of our concern are:

$$
C_{\mathrm{ad}}^{2}(H ; H)=\operatorname{Hom}\left(H^{\otimes 2}, H\right), \quad C_{\mathrm{ad}}^{3}(H ; H)=\operatorname{Hom}\left(H^{\otimes 3}, H\right) \oplus \operatorname{Hom}\left(H^{\otimes 2}, H^{\otimes 2}\right)
$$

For $n=1$, define

$$
C_{\text {ad }}^{1}(H ; H)=\left\{f \in \operatorname{Hom}_{k}(H, H) \mid f \mu=\mu(f \otimes 1)+\mu(1 \otimes f), \Delta f=(f \otimes 1) \Delta+(1 \otimes f) \Delta\right\}
$$

In the remaining sections we will define differentials that are homomorphisms between the chain groups:

$$
d^{n, i}: C_{\mathrm{ad}}^{n}(H ; H) \rightarrow C_{\mathrm{ad}}^{n+1, i}(H ; H)\left(=\operatorname{Hom}\left(H^{\otimes(n+2-i)}, H^{\otimes i}\right)\right)
$$

that will be defined individually for $n=1,2,3$ and for $i$ with $2 i \leq n+1$, and

$$
\begin{aligned}
& D_{1}=d^{1,1}: C_{\mathrm{ad}}^{1}(H ; H) \rightarrow C_{\mathrm{ad}}^{2}(H ; H), \quad D_{2}=d^{2,1}+d^{2,2}: C_{\mathrm{ad}}^{2}(H ; H) \rightarrow C_{\mathrm{ad}}^{3}(H ; H) \\
& D_{3}=d^{3,1}+d^{3,2}+d^{3,3}: C_{\mathrm{ad}}^{3}(H ; H) \rightarrow C_{\mathrm{ad}}^{3}(H ; H)
\end{aligned}
$$

\subsection{First differentials}

By analogy with the differential for multiplication, we make the following definition.

Definition 4. The first differential $d^{1,1}: C_{\text {ad }}^{1}(H ; H) \rightarrow C_{\text {ad }}^{2,1}(H ; H)$ is defined by

$$
d^{1,1}(f)=\operatorname{ad}(1 \otimes f)-f \operatorname{ad}+\operatorname{ad}(f \otimes 1)
$$

Diagrammatically, we represent $d^{1,1}$ as depicted in Fig. 7, where a 1-cochain is represented by a circle on a string.

$$
\mathrm{d}^{1,1}(\phi)=\hat{\phi}-\AA+\phi
$$

Figure 7: The 1-differential

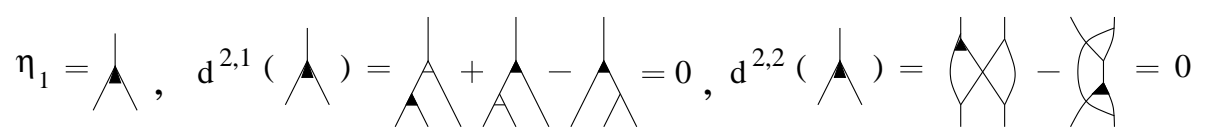

Figure 8: A diagram for a 2-cochain and the 2-cocycle conditions

\subsection{Second differentials}

Definition 5. Define the second differentials by $d_{\mathrm{ad}}^{2,1}(\phi)=\operatorname{ad}(\phi \otimes 1)+\phi(\operatorname{ad} \otimes 1)-\phi(1 \otimes \mu)$ and $d_{\mathrm{ad}}^{2,2}(\phi)=(\phi \otimes \mu)(1 \otimes \tau \otimes 1)(\Delta \otimes \Delta)-(1 \otimes \mu)(\tau \otimes 1)(1 \otimes \Delta)(1 \otimes \phi)(\tau \otimes 1)(1 \otimes \Delta)$

Diagrams for 2-cochain and 2-differentials are depicted in Fig. 8.

Theorem 1. $D_{2} D_{1}=0$.

Proof. This follows from direct calculations, and can be seen from diagrams in Figs. 9 and 10. 


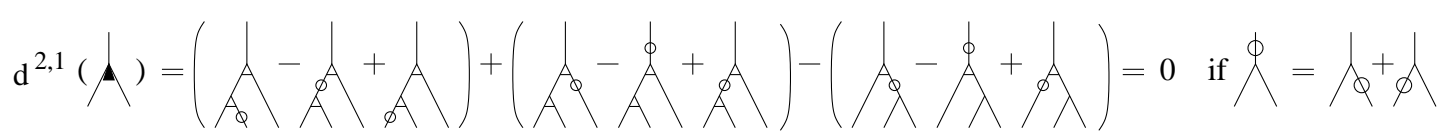

Figure 9: The 2-cocycle condition for a 2-coboundary, Part I

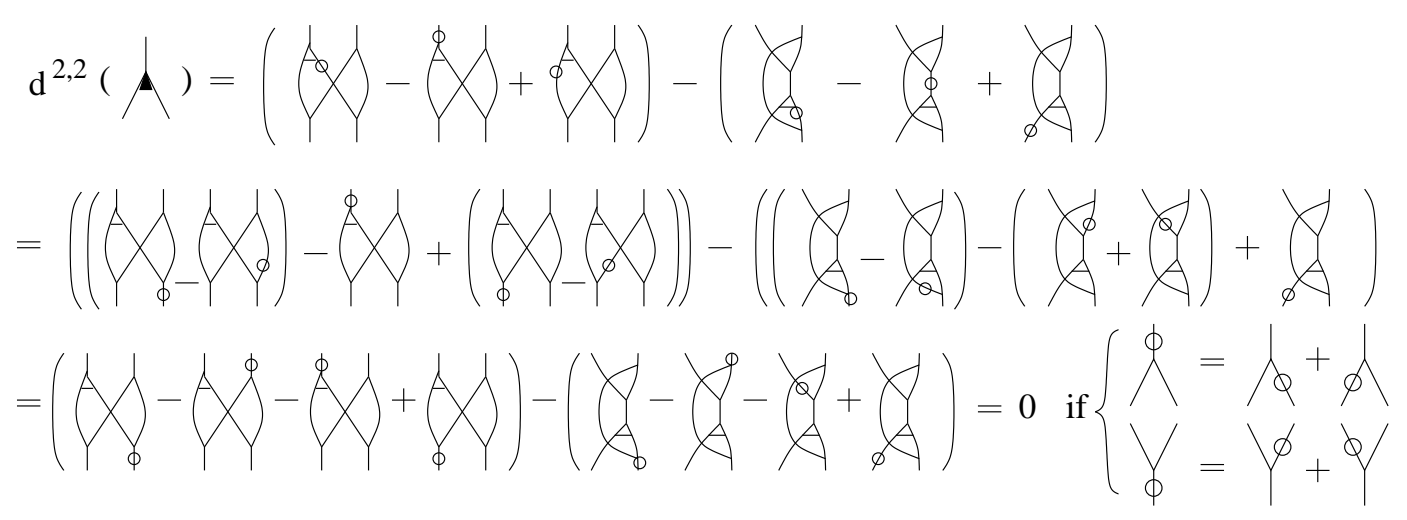

Figure 10: The 2-cocycle condition for a 2-coboundary, Part II

\subsection{Third differentials}

Definition 6. We define 3-differentials as follows. Let $\xi_{i} \in C^{3, i}(H ; H)$ for $i=1,2$. Then

$$
\begin{aligned}
d_{\mathrm{ad}}^{3,1}\left(\xi_{1}, \xi_{2}\right)= & \mathrm{ad}\left(\xi_{1} \otimes 1\right)+\xi_{1}(1 \otimes \mu \otimes 1)-\xi_{1}\left(\mathrm{ad} \otimes 1^{2}+1^{2} \otimes \mu\right) \\
d_{\mathrm{ad}}^{3,2}\left(\xi_{1}, \xi_{2}\right)= & (\mathrm{ad} \otimes \mu)(1 \otimes \tau \otimes 1)\left(1^{2} \otimes \Delta\right)\left(\xi_{2} \otimes 1\right)+(1 \otimes \mu)(\tau \otimes 1)\left(1 \otimes \xi_{2}\right)\left(R_{\mathrm{ad}} \otimes 1\right) \\
& +(1 \otimes \mu)\left(1^{2} \otimes \mu\right)\left(\tau \otimes 1^{2}\right)(1 \otimes \tau \otimes 1)\left(1^{2} \otimes \Delta\right)\left(\left(1^{2} \otimes \xi_{1}\right)\right. \\
& \left.\cdot\left(1 \otimes \tau \otimes 1^{2}\right)\left(\tau \otimes 1^{3}\right)\left(1^{2} \otimes \tau \otimes 1\right)(1 \otimes \Delta \otimes \Delta)\right) \\
& -\left(\xi_{1} \otimes \mu\right)\left(1^{2} \otimes \tau \otimes 1\right)\left(1^{2} \otimes \mu \otimes 1^{2}\right)\left(1 \otimes \tau \otimes 1^{3}\right)(\Delta \otimes \Delta \otimes \Delta)-\xi_{2}(1 \otimes \mu) \\
d_{\mathrm{ad}}^{3,3}\left(\xi_{1}, \xi_{2}\right)= & (1 \otimes \mu \otimes 1)\left(\tau \otimes 1^{2}\right)(1 \otimes \Delta \otimes 1)\left(1 \otimes \xi_{2}\right)(\tau \otimes 1)(1 \otimes \Delta) \\
& +\left(\xi_{2} \otimes \mu\right)(1 \otimes \tau \otimes 1)(\Delta \otimes \Delta)-(1 \otimes \Delta) \xi_{2}
\end{aligned}
$$

Diagrams for 3-cochains are depicted in Fig. 11. See Fig. 12 (A), (B), and (C) for the diagrammatics for $d^{3,1}, d^{3,2}$ and $d^{3,3}$, respectively.

Theorem 2. $D_{3} D_{2}=0$.

Proof. The proof follows from direct calculations that are indicated in Figs. 13, 14 and 15. We demonstrate how to recover algebraic calculations from these diagrams for the part $\left(d^{3,3} d^{2,2}\right)\left(\eta_{1}\right)=0$ for any $\eta_{1} \in C^{2}(H ; H)$. This is indicated in Fig. 15, where subscripts ad are suppressed for simplicity. Let $\xi_{2}=d^{2,2}\left(\eta_{1}\right) \in C^{3,2}(H ; H)$ (note that $\xi_{1}=d^{2,1}\left(\eta_{1}\right) \in C^{3,1}(H ; H)$ does not land in the domain of $\left.d^{3,3}\right)$. The first line of Fig. 15 represents the definition of the

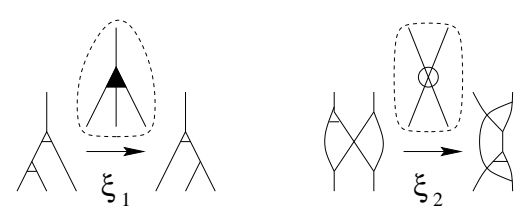

Figure 11: Diagrams for 3-cochains 


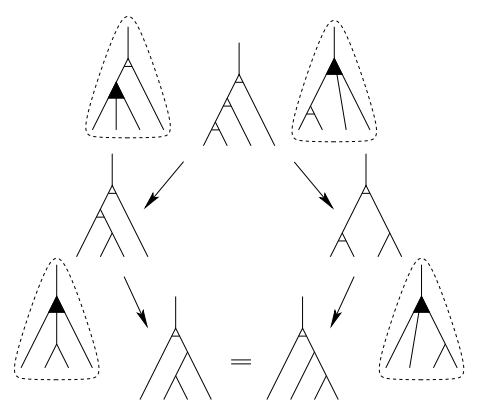

(A)

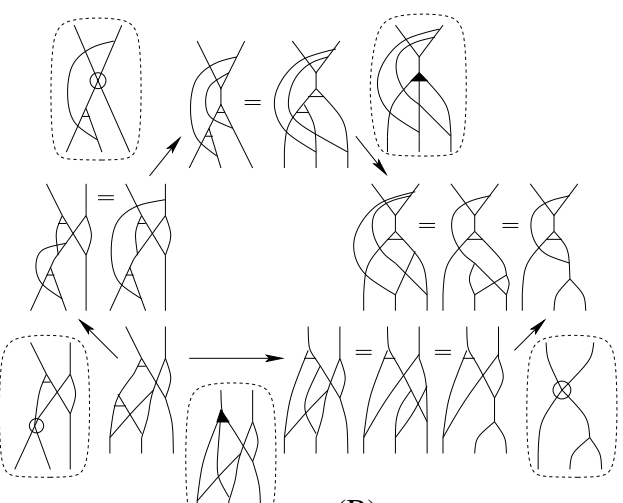

(B)

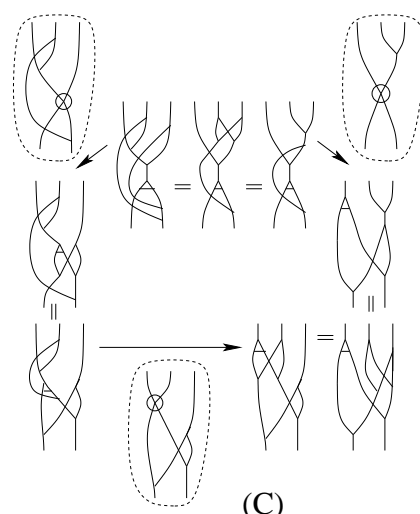

(C)

Figure 12: The 3-differentials

differential

$$
\begin{aligned}
d_{\mathrm{ad}}^{3,3}\left(\xi_{1}, \xi_{2}\right)= & (1 \otimes \mu \otimes 1)\left(\tau \otimes 1^{2}\right)(1 \otimes \Delta \otimes 1)\left(1 \otimes \xi_{2}\right)(\tau \otimes 1)(1 \otimes \Delta) \\
& +\left(\xi_{2} \otimes \mu\right)(1 \otimes \tau \otimes 1)(\Delta \otimes \Delta)-(1 \otimes \Delta) \xi_{2}
\end{aligned}
$$

where each term represents each connected diagram. The first parenthesis of the second line represents that

$$
\xi_{2}=d^{2,2}\left(\eta_{1}\right)=\left(\eta_{1} \otimes \mu\right)(1 \otimes \tau \otimes 1)(\Delta \otimes \Delta)-(1 \otimes \mu)(\tau \otimes 1)(1 \otimes \Delta)\left(1 \otimes \eta_{1}\right)(\tau \otimes 1)(1 \otimes \Delta)
$$

is substituted in the first term $(1 \otimes \mu \otimes 1)\left(\tau \otimes 1^{2}\right)(1 \otimes \Delta \otimes 1)\left(1 \otimes \xi_{2}\right)(\tau \otimes 1)(1 \otimes \Delta)$. When these two maps are applied to a general element $x \otimes y \in H \otimes H$, the results are computed as

$$
\begin{aligned}
& \eta_{1}\left(x_{(1)} \otimes y_{(2)(1)}\right)_{(1)} \otimes y_{(1)} \eta_{1}\left(x_{(1)} \otimes y_{(2)(1)}\right)_{(2)} \otimes x_{(2)} y_{(2)(2)} \\
& -\eta_{1}\left(x \otimes y_{(2)(2)}\right)_{(1)(1)} \otimes y_{(1)} \eta_{1}\left(x \otimes y_{(2)(2)}\right)_{(1)(2)} \otimes y_{(2)(1)} \eta_{1}\left(x \otimes y_{(2)(2)}\right)_{(2)}
\end{aligned}
$$

By coassociativity applied to $y$ and $\eta_{1}\left(x \otimes y_{(2)(2)}\right)$, the second term is equal to

$$
\eta_{1}\left(x \otimes y_{(2)}\right)_{(1)} \otimes y_{(1)(1)} \eta_{1}\left(x \otimes y_{(2)}\right)_{(2)(1)} \otimes y_{(1)(2)} \eta_{1}\left(x \otimes y_{(2)}\right)_{(2)(2)}
$$

which is equal, by compatibility, to $\eta_{1}\left(x \otimes y_{(2)}\right)_{(1)} \otimes\left(y_{(1)} \eta_{1}\left(x \otimes y_{(2)}\right)_{(2)}\right)_{(1)} \otimes\left(y_{(1)} \eta_{1}\left(x \otimes y_{(2)}\right)_{(2)}\right)_{(2)}$. This last term is represented exactly by the last term in the second line of Fig. 15, and therefore is cancelled. The map represented by the second term in the second line of Fig. 15 cancels with the third term by coassociativity, and the fourth term cancels with the sixth by coassociativity applied twice and compatibility once. Other cases (Figs. 13, 14) are computed similarly.

From point of view of Lemma 2, we state the relation between deformations and the third differential map as follows.

Corollary 1. The primary obstructions $\left(\xi_{1}, \xi_{2}\right)$ to formal deformations in Lemma 2 represent a 3-cocycle: $D_{3}\left(\xi_{1}, \xi_{2}\right)=0$.

Furthermore, in Lemma 2 we see that $D_{2}\left(\operatorname{ad}_{n+1}\right)=\left(\xi_{1}, \xi_{2}\right)$, so we regard that the obstructions represent a cohomology class $\left[\xi_{1}, \xi_{2}\right] \in H^{3}(H ; H)$ after the definition of the cohomology groups in the next section. 


\subsection{Cohomology groups}

For convenience define $C^{0}(H ; H)=0, D_{0}=0: C^{0}(H ; H) \rightarrow C^{1}(H ; H)$.

Then Theorems 1 and 2 are summarized as follows.

Theorem 3. $\mathcal{C}=\left(C^{n}, D_{n}\right)_{n=0,1,2,3}$ is a chain complex.

This enables us to propose

Definition 7. The adjoint $n$-coboundary, cocycle and cohomology groups are defined by:

$$
B^{n}(H ; H)=\operatorname{Im}\left(D_{n-1}\right), \quad Z^{n}(H ; H)=\operatorname{Ker}\left(D_{n}\right), \quad H^{n}(H ; H)=Z^{n}(H ; H) / B^{n}(H ; H)
$$

for $n=1,2,3$.

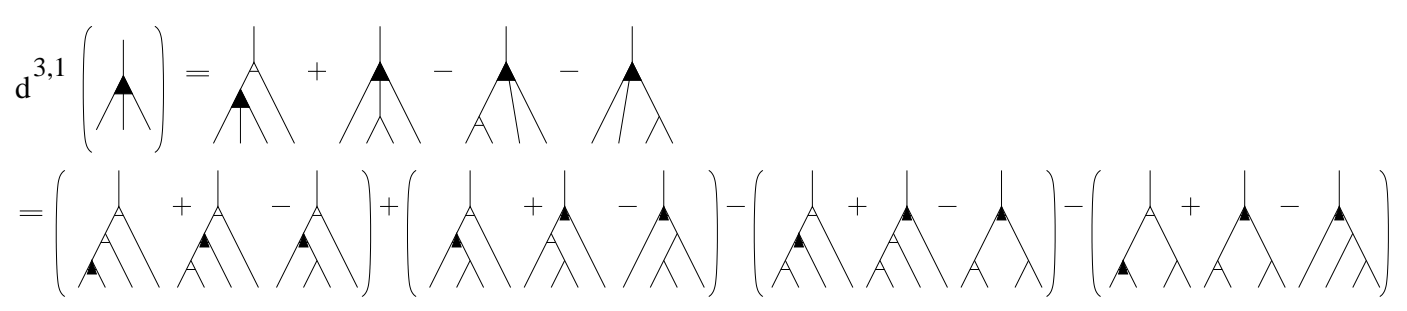

Figure 13: $d^{3,1}\left(d^{2,1}\right)=0$

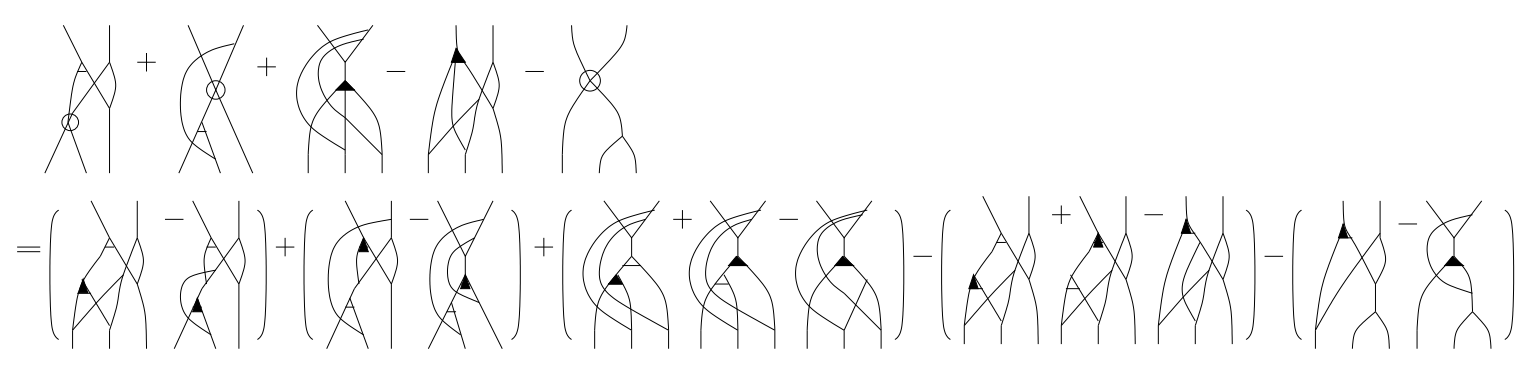

Figure 14: $d^{3,2}\left(d^{2,1}, d^{2,2}\right)=0$

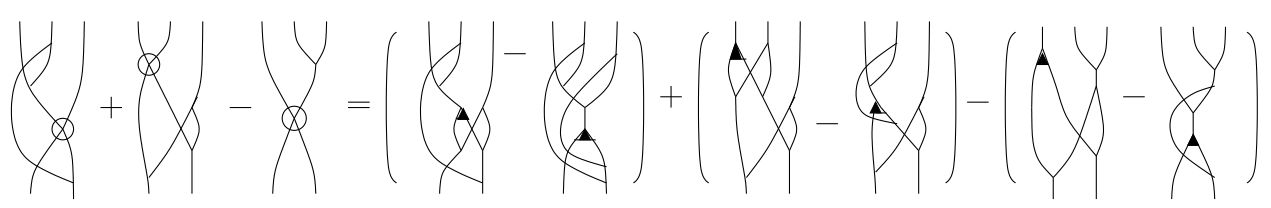

Figure 15: $d^{3,3}\left(d^{2,2}\right)=0$

\section{Examples}

\subsection{Group algebras}

Let $G$ be a group and $H=k G$ be its group algebra with the coefficient field $k$ (char $k \neq 2$ ). Then $H$ has a Hopf algebra structure induced from the group operation as multiplication, $\Delta(x)=x \otimes x$ for basis elements $x \in G$, and the antipode induced from $S(x)=x^{-1}$ for $x \in G$. Here and below, we denote the conjugation action on a group $G$ by $x \triangleleft y:=y^{-1} x y$. Note that this defines a quandle structure on $G$; see [12]. 
Lemma 3. $C_{\text {ad }}^{1}(k G ; k G)=0$.

Proof. For any given $w \in G$ write $f(w)=\sum_{u \in G} a_{u}(w) u$, where $a: G \rightarrow k$ is a function. Recall the defining equality (4.1). The LHS of the second condition is written as

$$
\Delta f(w)=\Delta\left(\sum_{u} a_{u}(w) u\right)=\sum_{u} a_{u}(w) u \otimes u
$$

and the RHS is written as

$$
((f \otimes 1) \Delta+(1 \otimes f) \Delta)(w)=((f \otimes 1)+(1 \otimes f))(w \otimes w)=\sum_{h} a_{h}(w)(h \otimes w)+\sum_{v} a_{v}(w)(w \otimes v)
$$

For a given $w$, fix $u$ and then compare the coefficients of $u \otimes u$. In the LHS we have $a_{u}(w)$, while on the RHS $w=u$, and furthermore $w=h=v$ for $u \otimes u$. Thus the diagonal coefficient must satisfy $a_{w}(w)=a_{w}(w)+a_{w}(w)$, so that $a_{w}(w)=0$ since char $k \neq 2$. In the case $w \neq u$, neither term of $h \otimes w$ nor $w \otimes v$ is equal to $u \otimes u$, hence $a_{u}(w)=0$.

Lemma 4. For $x, y \in G$, write $\phi(x, y)=\sum_{u} a_{u}(x, y) u$, where $a: G \times G \rightarrow k$. Then the induced linear map $\phi: k G \otimes k G \rightarrow k G$ is in $Z_{\mathrm{ad}}^{2}(k G ; k G)$ if and only if a satisfies

$$
a_{x \triangleleft y}(x, y)+a_{(x \triangleleft y) \triangleleft z}(x \triangleleft y, z)-a_{(x \triangleleft y) \triangleleft z}(x, y z)=0
$$

for any $x, y, z \in G$.

Proof. The first 2-cocycle condition for $\phi: k G \otimes k G \rightarrow k G$ is written by

$$
z^{-1} \phi(x \otimes y) z+\phi\left(y^{-1} x y \otimes z\right)-\phi(x \otimes y z)=0
$$

for basis elements $x, y, z \in G$. The second is formulated by

$$
\mathrm{LHS}=\phi(x \otimes y) \otimes x y=\sum_{u} a_{u}(x, y)(u \otimes x y), \quad \text { RHS }=\sum_{w} a_{w}(x, y)(w \otimes y w)
$$

They have the common term $u \otimes x y$ for $w=y^{-1} x y=u$, and otherwise they are different terms. Thus we obtain $a_{w}(x, y)=0$ unless $w=y^{-1} x y$. For these terms, the first condition becomes

$$
z^{-1}\left(a_{y^{-1} x y}(x, y) y^{-1} x y\right) z+a_{z^{-1} y^{-1} x y z}\left(y^{-1} x y, z\right) z^{-1} y^{-1} x y z-a_{z^{-1} y^{-1} x y z}(x, y z) z^{-1} y^{-1} x y z=0
$$

and the result follows.

Remark 3. In the preceding proof, since the term $a_{w}(x, y)=0$ unless $w=x \triangleleft y$, let $a_{x \triangleleft y}(x, y)=$ $a(x, y)$. Then the condition stated becomes $a(x, y)+a(x \triangleleft y, z)-a(x, y z)=0$.

Proposition 1. Let $G$ be a group. Let $\left(\xi_{1}, \xi_{2}\right) \in C_{\mathrm{ad}}^{3}(k G ; k G)$, where $\xi_{1}$ is the map that is defined by linearly extending $\xi_{1}(x \otimes y \otimes z)=\sum_{u \in G} c_{u}(x, y, z) u$. Then $\left(\xi_{1}, \xi_{2}\right) \in Z_{\text {ad }}^{3}(k G ; k G)$ if and only if $\xi_{2}=0$ and the coefficients satisfy the following properties: (a) $c_{u}(x, y, z)=0$ if $u \neq z^{-1} y^{-1} x y z$, and $(\mathrm{b}) c(x, y, z)=c_{z^{-1} y^{-1} x y z}(x, y, z)$ satisfies

$$
c(x, y, z)+c(x, y z, w)=c\left(y^{-1} x y, z, w\right)+c(x, y, z w)
$$

Proof. Suppose $\left(\xi_{1}, \xi_{2}\right) \in Z_{\text {ad }}^{3}(k G ; k G)$. Let $\xi_{2}$ be the map that is defined by linearly extending $\xi_{2}(x \otimes y)=\sum_{u, v \in G} a_{u, v}(x, y) u \otimes v$. Then the third 3-cocycle condition from Definition 6 gives: (abbreviating $a_{u, v}(x, y)=a_{u, v}$ )

$$
d^{3,3}\left(\xi_{1}, \xi_{2}\right)(x \otimes y)=\sum_{u_{1}, v_{1}} a_{u_{1}, v_{1}}\left(u_{1} \otimes y u_{1} \otimes v_{1}\right)+\sum_{u_{2}, v_{2}} a_{u_{2}, v_{2}}\left(u_{2} \otimes v_{2} \otimes x y\right)
$$




$$
-\sum_{u_{3}, v_{3}} a_{u_{3}, v_{3}}\left(u_{3} \otimes v_{3} \otimes v_{3}\right)=0
$$

We first consider terms in which the third tensorand is $x y$. From the third summand, this forces the second tensorand to be $x y$, so we collect the terms of the form $(u \otimes x y \otimes x y)$. This gives

$$
\sum_{u}\left(a_{u, x y}+a_{u, x y}-a_{u, x y}\right)(u \otimes x y \otimes x y)=0
$$

which implies $a_{u, x y}=0$ for all $u \in G$. The remaining terms are

$$
\sum_{u_{1}, v_{1} \neq x y} a_{u_{1}, v_{1}}\left(u_{1} \otimes y u_{1} \otimes v_{1}\right)+\sum_{u_{2}, v_{2} \neq x y} a_{u_{2}, v_{2}}\left(u_{2} \otimes v_{2} \otimes x y\right)-\sum_{u_{3}, v_{3} \neq x y} a_{u_{3}, v_{3}}\left(u_{3} \otimes v_{3} \otimes v_{3}\right)=0
$$

From the second sum we obtain $a_{u, v}(x, y)=0$ for $v \neq x y$. In conclusion, if $d^{3,3}\left(\xi_{1}, \xi_{2}\right)=0$ for $k G$ then $\xi_{2}=0$.

We now consider $d^{3,2}\left(\xi_{1}, \xi_{2}\right)$, with $\xi_{2}=0$. Let $\xi_{1}$ be the map that is defined by linearly extending $\xi_{1}(x \otimes y \otimes z)=\sum_{u \in G} c_{u}(x, y, z) u$ for $x, y, z \in G$. The second 3-cocycle condition from Definition 6, with $\xi_{2}=0$, is $\sum_{u} c_{u} u \otimes y z u=\sum_{v} c_{v} v \otimes x y z$. In order to combine like terms, we need $y z u=x y z$, meaning $u=z^{-1} y^{-1} x y z$. Thus, $c_{u}(x, y, z)=0$ except in the case when $u=z^{-1} y^{-1} x y z$. In this case, we obtain $\xi_{1}(x \otimes y \otimes z)=c(x, y, z) z^{-1} y^{-1} x y z \otimes x y z$, where $c(x, y, z)=c_{z^{-1} y^{-1} x y z}(x, y, z)$.

Finally we consider the first 3-cocycle condition from Definition 6, which is formulated for basis elements by

$$
w^{-1} \xi_{1}(x \otimes y \otimes z) w+\xi_{1}(x \otimes y z \otimes w)=\xi_{1}(x \triangleleft y \otimes z \otimes w)+\xi_{1}(x \otimes y \otimes z w)
$$

Substituting in the formula for $c(x, y, z)$ which we found above, we obtain

$$
c(x, y, z)+c(x, y z, w)=c\left(y^{-1} x y, z, w\right)+c(x, y, z w)
$$

This is a group 3-cocycle condition with the first term $x \cdot c(y, z, w)$ omitted. This is expected from Fig. 12 (A). Constant functions, for example, satisfy this condition.

Next we look at a coboundary condition. A 3-coboundary is written as

$$
\xi_{1}(x \otimes y \otimes z)=\sum_{u} c_{u}(x, y, z) u=d^{2,1}(\phi)(x \otimes y \otimes z)=z^{-1} \phi(x \otimes y) z+\phi\left(y^{-1} x y \otimes z\right)-\phi(x \otimes y z)
$$

If we write $\phi(x, y)=\sum_{u} h_{u}(x, y) u$, then

$$
\begin{aligned}
\left(d^{2,1}(\phi)\right)(x \otimes y \otimes z) & =z^{-1}\left(\sum_{u} h_{u}(x, y) u\right) z+\left(\sum_{v} h_{v}\left(y^{-1} x y, z\right) v\right)-\left(\sum_{w} h_{w}(x, y z) w\right) \\
& =\sum_{g}\left(h_{z g z^{-1}}(x, y)+h_{g}\left(y^{-1} x y, z\right)-h_{g}(x, y z)\right) g
\end{aligned}
$$

Hence

$$
c_{u}(x, y, z)=h_{z u z^{-1}}(x, y)+h_{u}\left(y^{-1} x y, z\right)-h_{u}(x, y z)
$$

and in particular for the coefficients $c_{u}(x, y, z)$ from Proposition 1 ,

$$
c(x, y, z)=c_{z^{-1} y^{-1} x y z}(x, y, z)=h_{y^{-1} x y}(x, y)+h_{z^{-1} y^{-1} x y z}\left(y^{-1} x y, z\right)-h_{z^{-1} y^{-1} x y z}(x, y z)
$$

By setting $h_{y^{-1} x y}(x, y)=a(x, y)$, we obtain 
Lemma 5. A 3-cocycle $c(x, y, z)$ is a coboundary if for some $a(x, y)$,

$$
c(x, y, z)=a(x, y)+a\left(y^{-1} x y, z\right)-a(x, y z)
$$

Remark 4. From Remark 3, Proposition 1, and Lemma 5, we have the following situation. The 2 -cocycle condition, the 3 -cocycle condition, and the 3-coboundary condition, respectively, gives rise to the equations

$$
\begin{aligned}
& a(x, y)+a\left(y^{-1} x y, z\right)-a(x, y z)=0 \\
& c(x, y, z)+c(x, y z, w)-c\left(y^{-1} x y, z, w\right)-c(x, y, z w)=0 \\
& c(x, y, z)=a(x, y)+a\left(y^{-1} x y, z\right)-a(x, y z)
\end{aligned}
$$

This suggests a cohomology theory, which we investigate in Section 6.

Proposition 2. For the symmetric group $G=S_{3}$ on three letters, we have $H_{\text {ad }}^{1}(k G ; k G)=0$ and $H_{\text {ad }}^{2}(k G ; k G) \cong \bigoplus_{3}(k G)$ for $k=\mathbb{C}$ and $\mathbb{F}_{3}$.

Proof. By Lemma 3, we have $H_{\mathrm{ad}}^{1}(k G ; k G)=0$ and $B_{\text {ad }}^{2}(k G, k G)=0$. Hence $H^{2}(k G ; k G) \cong$ $Z_{\text {ad }}^{2}(k G ; k G)$, which is computed by solving the system of equations stated in Lemma 4 and Remark 3. Computations by Maple and Mathematica shows that the solution set is of dimension 3 and generated by $\left(a\left(\left(\begin{array}{lll}1 & 2 & 3\end{array}\right),\left(\begin{array}{ll}1 & 2\end{array}\right)\right), a\left(\left(\begin{array}{ll}2 & 3\end{array}\right),\left(\begin{array}{ll}1 & 3\end{array}\right)\right)\right.$, and $a\left(\left(\begin{array}{ll}1 & 3\end{array}\right),\left(\begin{array}{l}1 \\ 2\end{array}\right)\right)$ for the above mentioned coefficient fields.

\subsection{Function algebras on groups}

Let $G$ be a finite group and $k$ a field with $\operatorname{char}(k) \neq 2$. The set $k^{G}$ of functions from $G$ to $k$ with pointwise addition and multiplication is a unital associative algebra. It has a Hopf algebra structure using $k^{G \times G} \cong k^{G} \otimes k^{G}$ with comultiplication defined through $\Delta: k^{G} \rightarrow k^{G \times G}$ by $\Delta(f)(u \otimes v)=f(u v)$ and the antipode by $S(f)(x)=f\left(x^{-1}\right)$.

Now $k^{G}$ has basis (the characteristic function) $\delta_{g}: G \rightarrow k$ defined by $\delta_{g}(x)=1$ if $x=g$ and zero otherwise. Since $S\left(\delta_{g}\right)=\delta_{g^{-1}}$ and $\Delta\left(\delta_{h}\right)=\sum_{u v=h} \delta_{u} \otimes \delta_{v}$, the adjoint map becomes

$$
\operatorname{ad}\left(\delta_{g} \otimes \delta_{h}\right)=\sum_{u v=h} \delta_{u^{-1}} \delta_{g} \delta_{v}= \begin{cases}\delta_{g} & \text { if } h=1 \\ 0 & \text { otherwise }\end{cases}
$$

Lemma 6. $C_{\text {ad }}^{1}\left(k^{G} ; k^{G}\right)=0$.

Proof. Recall the defining equality (4.1). Let $G=\left\{g_{1}, \ldots, g_{n}\right\}$ be a given finite group and abbreviate $\delta_{g_{i}}=\delta_{i}$ for $i=1, \ldots, n$. Describe $f: k^{G} \rightarrow k^{G}$ by $f\left(\delta_{i}\right)=\sum_{j=1}^{n} s_{i}^{j} \delta_{j}$. Then $f \mu=\mu(f \otimes 1)+\mu(1 \otimes f)$ is written for basis elements by LHS $=f\left(\delta_{i} \delta_{j}\right)$ and

$$
\mathrm{RHS}=f\left(\delta_{i}\right) \delta_{j}+\delta_{i} f\left(\delta_{j}\right)=\left(\sum_{\ell=1}^{n} s_{i}^{\ell} \delta_{\ell}\right) \delta_{j}+\delta_{i}\left(\sum_{h=1}^{n} s_{j}^{h} \delta_{h}\right)=s_{i}^{j} \delta_{j}+s_{j}^{i} \delta_{i}
$$

For $i=j$ we obtain LHS $=\sum_{w=1}^{n} s_{i}^{w} \delta_{w}$ and RHS $=2 s_{i}^{i} \delta_{i}$ so that $s_{i}^{j}=0$ for all $i, j$ as desired.

Lemma 7. $Z_{\text {ad }}^{2}\left(k^{G} ; k^{G}\right)=0$.

Proof. Recall that $d^{2,1}\left(\eta_{1}\right)=\operatorname{ad}\left(\eta_{1} \otimes 1\right)+\eta_{1}(\operatorname{ad} \otimes 1)-\eta_{1}(1 \otimes \mu)$ for $\eta_{1} \in C_{\text {ad }}^{2}\left(k^{G}, k^{G}\right)$. Describe a general element $\eta_{1} \in C_{\text {ad }}^{2}\left(k^{G}, k^{G}\right)$ by $\eta_{1}\left(\delta_{i} \otimes \delta_{j}\right)=\sum_{\ell} s_{i j}^{\ell} \delta_{\ell}$. Consider $d^{2,1}\left(\eta_{1}\right)\left(\delta_{a} \otimes \delta_{b} \otimes \delta_{c}\right)$. If $c \neq 1$, then the first term is zero by the definition of ad. If $c \neq 1$ and $b=1$, then the third term is also zero, and we obtain that the second term $\eta_{1}\left(\delta_{a} \otimes \delta_{c}\right)$ is zero. Hence $\eta_{1}\left(\delta_{a} \otimes \delta_{c}\right)=0$ unless $c=1$. Next, set $b=c=1$ in the general form. Then all three terms equal $\eta_{1}\left(\delta_{a} \otimes \delta_{1}\right)$ and we obtain $\eta_{1}\left(\delta_{a} \otimes \delta_{1}\right)=0$, and the result follows. 
By combining the above lemmas, we obtain the following

Theorem 4. For any finite group $G$ and a field $k$, we have $H_{\mathrm{ad}}^{n}\left(k^{G} ; k^{G}\right)=0$ for $n=1,2$.

Observe that $k(G)$ and $k^{G}$ are cohomologically distinct.

\subsection{Bosonization of the superline}

Let $H$ be generated by $1, g, x$ with relations $x^{2}=0, g^{2}=1, x g=-g x$ and Hopf algebra structure $\Delta(x)=x \otimes 1+g \otimes x, \Delta(g)=g \otimes g, \epsilon(x)=0, \epsilon(g)=1, S(x)=-g x, S(g)=g$ (this Hopf algebra is called the bosonization of the superline [15], page 39, Example 2.1.7).

The operation ad is represented by the following table, where, for example, $\operatorname{ad}(g \otimes x)=2 x$.

\begin{tabular}{|r||r|r|r|r|}
\hline & 1 & $g$ & $x$ & $g x$ \\
\hline \hline 1 & 1 & 1 & 0 & 0 \\
\hline$g$ & $g$ & $g$ & $2 x$ & $2 x$ \\
\hline$x$ & $x$ & $-x$ & 0 & 0 \\
\hline$g x$ & $g x$ & $-g x$ & 0 & 0 \\
\hline
\end{tabular}

Remark 5. The induced $R$-matrix $R_{\text {ad }}$ has determinant 1 , the characteristic polynomial is $\left(\lambda^{2}+1\right)^{2}(\lambda+1)^{4}(\lambda-1)^{8}$, and the minimal polynomial is $\left(\lambda^{2}+1\right)(\lambda+1)(\lambda-1)^{2}$.

Proposition 3. The first cohomology of $H$ is given by $H_{\mathrm{ad}}^{1}(H, H) \cong k$.

Proof. Recall the defining equality (4.1). Let $f \in C_{\text {ad }}^{1}(H ; H)$. Assume that $f(x)=a+b x+$ $c g+d x g$ and $f(g)=\alpha+\beta x+\gamma g+\delta x g$ where $a, b, c, d, \alpha, \beta, \gamma, \delta \in k$. Applying $f$ to both sides of the equation $g^{2}=1$, one obtains $\alpha=\gamma=0$. Similarly evaluating both sides of the equation $\Delta f=(f \otimes 1) \Delta+(1 \otimes f) \Delta$ at $g$ gives $\beta=\delta=0$, one obtains that $f(g)=0$. In a similar way, applying $f$ to the equations $x^{2}=0$ and $x g=-g x$ gives rise to, respectively, $a=0$ and $c=0$. Also evaluating $\Delta f=(f \otimes 1) \Delta+(1 \otimes f) \Delta$ at $x$ gives rise to $d=0$. We also have $f(x)=f(x g) g$ (since $g^{2}=1$ ), which implies $f(x g)=b x g$. In conclusion $f$ satisfies $f(1)=0=f(g), f(x)=b x$, and $f(x g)=b(x g)$. Now consider $f$ in the kernel of $D_{1}$, that is $f$ satisfies

$$
d^{1,1}(f)=\operatorname{ad}(1 \otimes f)-f \operatorname{ad}+\operatorname{ad}(f \otimes 1)
$$

It is directly checked on all the generators $u \otimes v$ of $H \otimes H$ that $d^{1,1}(f)(u \otimes v)=0$. This implies that $H^{1}(H, H) \cong k$.

Proposition 4. For any field $k$ of characteristic not $2, H_{\mathrm{ad}}^{2}(H, H) \cong k^{3}$.

Proof. With $d^{1,1}=0$ from the preceding Proposition, we have $H_{\mathrm{ad}}^{2}(H, H) \cong Z_{\mathrm{ad}}^{2}(H, H)$. Either the direct hand calculations from definitions or the computer calculations give the following general solution for the 2-cocycle $\phi(a \otimes b)$ represented in the following table:

\begin{tabular}{|r||r|r|r|r|}
\hline & 1 & $g$ & $x$ & $g x$ \\
\hline \hline 1 & 0 & 0 & 0 & 0 \\
\hline$g$ & 0 & 0 & $\alpha 1$ & $\alpha 1$ \\
\hline$x$ & 0 & $-\alpha 1$ & 0 & 0 \\
\hline$g x$ & 0 & $\gamma g$ & $\beta 1+\gamma x$ & $-\beta 1+\gamma x$ \\
\hline
\end{tabular}

Here, for example, $\phi(g x \otimes g)=\gamma g$, where $\alpha, \beta, \gamma$ are free variables. 


\section{Adjoint, groupoid, and quandle cohomology theories}

From Remark 4, the adjoint cohomology leads us to cohomology, especially for conjugate groupoids of groups as defined below. Through the relation between Reidemeister moves for knots and the adjoint, groupoid cohomology, we obtain a new construction of quandle cocycles. In this section we investigate these relations. First we formulate a general definition. Many formulations of groupoid cohomology can be found in literature, and relations of the following formulation to previously known theories are not clear. See [20], for example.

Let $\mathcal{G}$ be a groupoid with objects $\mathrm{Ob}(\mathcal{G})$ and morphisms $G(x, y)$ for $x, y \in \mathrm{Ob}(\mathcal{G})$. Let $f_{i} \in G\left(x_{i}, x_{i+1}\right), 0 \leq i<n$, for non-negative integers $i$ and $n$. Let $C_{n}(\mathcal{G})$ be the free abelian group generated by $\left\{\left(x_{0}, f_{0}, \ldots, f_{n}\right) \mid x_{0} \in \mathrm{Ob}(\mathcal{G}), f_{i} \in G\left(x_{i}, x_{i+1}\right), 0 \leq i<n\right\}$. The boundary map $\partial: C_{n+1}(\mathcal{G}) \rightarrow C_{n}(\mathcal{G})$ is defined by by linearly extending

$$
\begin{aligned}
\partial\left(x_{0}, f_{0}, \ldots, f_{n}\right) & =\left(x_{1}, f_{1}, \ldots, f_{n}\right)+\sum_{i=0}^{n-1}(-1)^{i+1}\left(x_{0}, f_{0}, \ldots, f_{i-1}, f_{i} f_{i+1}, f_{i+2}, \ldots, f_{n}\right) \\
& +(-1)^{n+1}\left(x_{0}, f_{0} \ldots, f_{n-1}\right)
\end{aligned}
$$

Then it is easily seen that this differential defines a chain complex. The corresponding groupoid 1- and 2-cocycle conditions are written as

$$
\begin{aligned}
& a\left(x_{1}, f_{1}\right)-a\left(x_{0}, f_{0} f_{1}\right)+a\left(x_{0}, f_{0}\right)=0 \\
& c\left(x_{1}, f_{1}, f_{2}\right)-c\left(x_{0}, f_{0} f_{1}, f_{2}\right)+c\left(x_{0}, f_{0}, f_{1} f_{2}\right)-c\left(x_{0}, f_{0}, f_{1}\right)=0
\end{aligned}
$$

The general cohomological theory of homomorphisms and extensions applies, such as:

Remark 6. Let $\mathcal{G}$ be a groupoid and $A$ be an abelian group regarded as a one-object groupoid. Then $\alpha: \operatorname{hom}\left(x_{0}, x_{1}\right) \rightarrow A$ gives a groupoid homomorphism from $\mathcal{G}$ to $A$, which sends $\operatorname{Ob}(\mathcal{G})$ to the single object of $A$, if and only if $a: C_{1}(\mathcal{G}) \rightarrow A$, defined by $a\left(x_{0}, f_{0}\right)=\alpha\left(f_{0}\right)$, is a groupoid 1-cocycle. Next we consider extensions of groupoids. Define

$$
\circ:\left(\operatorname{hom}\left(x_{0}, x_{1}\right) \times A\right) \times\left(\operatorname{hom}\left(x_{1}, x_{2}\right) \times A\right) \rightarrow \operatorname{hom}\left(x_{0}, x_{2}\right) \times A
$$

by $\left(f_{0}, a\right) \circ\left(f_{1}, b\right)=\left(f_{0} f_{1}, a+b+c\left(x_{0}, f_{0}, f_{1}\right)\right)$, where $c\left(x_{0}, f_{0}, f_{1}\right) \in \operatorname{hom}\left(C_{2}(\mathcal{G}), A\right)$. If $\mathcal{G} \times A$ is a groupoid, the function $c$ with the value $c\left(x_{0}, f_{0}, f_{1}\right)$ is a groupoid 2-cocycle.

Example. Let $G$ be a group. Define the conjugate groupoid of $G$, denoted $\widehat{G}$, by $\operatorname{Ob}(\widehat{G})=G$ and $\operatorname{Mor}(\widehat{G})=G \times G$, where the source of the morphism $(x, y) \in \operatorname{hom}\left(x, y^{-1} x y\right)$ is $x$ and its target is $y^{-1} x y$, for $x, y \in G$. Composition is defined by $(x, y) \circ\left(y^{-1} x y, z\right)=(x, y z)$. For this example, the groupoid 1- and 2-cocycle conditions are

$$
\begin{aligned}
& a(x, y)+a\left(y^{-1} x y, z\right)-a(x, y z)=0 \\
& c(x, y, z)+c(x, y z, w)-c\left(y^{-1} x y, z, w\right)-c(x, y, z w)=0
\end{aligned}
$$

Diagrammatic representations of these equations are depicted in Fig. 16 (A) and (B), respectively. Furthermore, $c$ is a coboundary if

$$
c(x, y, z)=a(x, y)+a\left(y^{-1} x y, z\right)-a(x, y z)
$$

Compare with Remark 4.

For $G=\mathbb{S}_{3}$, the symmetric group on 3 letters, with coefficient group $\mathbb{C}, \mathbb{Z}_{2}, \mathbb{Z}_{3}, \mathbb{Z}_{5}$ and $\mathbb{Z}_{7}$, respectively, the dimensions of the conjugation groupoid 2-cocycles are $3,5,4,3$ and 3 . 
(A)

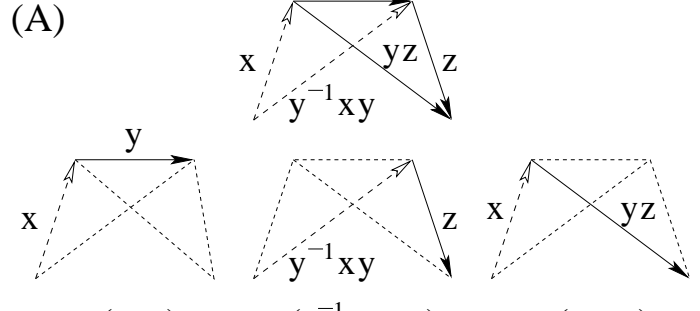

$\mathrm{a}(\mathrm{x}, \mathrm{y})+\mathrm{a}\left(\mathrm{y}^{-1} \mathrm{xy}, \mathrm{z}\right)=\mathrm{a}(\mathrm{x}, \mathrm{yz})$
(B)
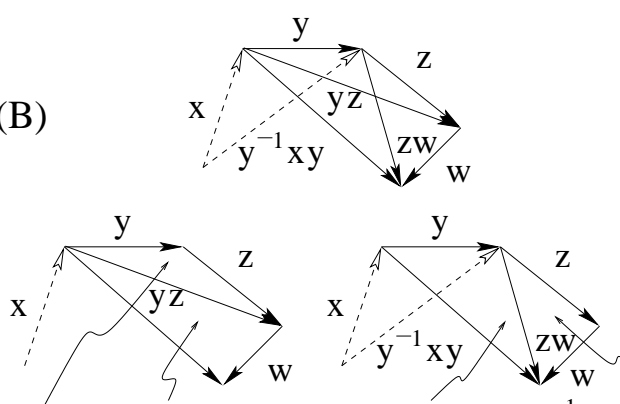

$c(x, y, z)+c(x, y z, w)=c(x, y, z w)+c\left(y^{-1} x y, z, w\right)$

Figure 16: Diagrams for groupoid 1- and 2-cocycles

For the rest of the section, we present new constructions of quandle cocycles from groupoid cocycles of conjugate groupoids of groups. Let $G$ be a finite group, and $a: G^{2} \rightarrow k$ be adjoint 2-cocycle coefficients that were defined in Remark 3 . These satisfy

$$
a(x, y)+a(x \triangleleft y, z)-a(x, y z)=0
$$

Proposition 5. Let $\psi(x, y)=a(x, y)$. Then $\psi$ satisfies the rack 2-cocycle condition

$$
\psi(x, y)+\psi(x \triangleleft y, z)=\psi(x, z)+\psi(x \triangleleft z, y \triangleleft z)
$$

Proof. By definition

$$
\begin{aligned}
& \psi(x, y)+\psi(x \triangleleft y, z)=a(x, y z) \\
& \psi(x, z)+\psi(x \triangleleft z, y \triangleleft z)=a(x, z)+a\left(z^{-1} x z, z^{-1} y z\right)=a(x, z(y \triangleleft z))
\end{aligned}
$$

Let $G$ be a finite group, and $c: G^{3} \rightarrow k$ be a coefficient of the adjoint 3-cocycle defined in Proposition 1. This satisfies

$$
c(x, y, z)+c(x, y z, w)=c(x \triangleleft y, z, w)+c(x, y, z w)
$$

Proposition 6. Let $G$ be a group that is considered as a quandle under conjugation. Then $\theta: G^{3} \rightarrow k$ defined by

$$
\theta(x, y, z)=c(x, y, z)-c\left(x, z, z^{-1} y z\right)
$$

is a rack 3-cocycle.

Proof. We must show that $\theta$ satsifies

$$
\theta(x, y, z)+\theta(x \triangleleft z, y \triangleleft z, w)+\theta(x, z, w)=\theta(x \triangleleft y, z, w)+\theta(x, y, w)+\theta(x \triangleleft w, y \triangleleft w, z \triangleleft w)
$$

We compute

$$
\begin{aligned}
\text { LHS } & -\operatorname{RHS}=\left[c(x, y, z)-c\left(x, z, z^{-1} y z\right)\right] \\
& +\left[c\left(z^{-1} x z, z^{-1} y z, w\right)-c\left(z^{-1} x z, w, w^{-1} z^{-1} y z w\right)\right]+\left[c(x, z, w)-c\left(x, w, w^{-1} z w\right)\right] \\
& -\left[c\left(y^{-1} x y, z, w\right)-c\left(y^{-1} x y, w, w^{-1} z w\right)\right]-\left[c(x, y, w)-c\left(x, w, w^{-1} y w\right)\right] \\
& -\left[c\left(w^{-1} x w, w^{-1} y w, w^{-1} z w\right)-c\left(w^{-1} x w, w^{-1} z w, w^{-1} z^{-1} y z w\right)\right] \\
& =\left[c(x, y, z)-c\left(y^{-1} x y, z, w\right)\right]-\left[c\left(x, z, z^{-1} y z\right)-c\left(z^{-1} x z, z^{-1} y z, w\right)\right] \\
& +\left[c(x, z, w)-c\left(z^{-1} x z, w, w^{-1} z^{-1} y z w\right)\right] \\
& -\left[c\left(x, w, w^{-1} z w\right)-c\left(w^{-1} x w, w^{-1} z w, w^{-1} z^{-1} y z w\right)\right] \\
& -\left[c(x, y, w)-c\left(y^{-1} x y, w, w^{-1} z w\right)\right]+\left[c\left(x, w, w^{-1} y w\right)-c\left(w^{-1} x w, w^{-1} y w, w^{-1} z w\right)\right] \\
& =[-c(x, y z, w)+c(x, y, z w)]-\left[-c\left(x, z z^{-1} y z, w\right)+c\left(x, z, z^{-1} y z w\right)\right] \\
& +\left[-c\left(x, z w, w^{-1} z^{-1} y z w\right)+c\left(x, z, w w^{-1} z^{-1} y z w\right)\right]+c\left(x, w, w^{-1} y w w^{-1} z w\right]=0
\end{aligned}
$$




\section{Deformations of $R$-matrices by adjoint 2-cocycles}

In this section we give, in an explicit form, deformations of $R$-matrices by 2-cocycles of the adjoint cohomology theory we developed in this paper. Let $H$ be a Hopf algebra and ad its adjoint map. In Section 3 a deformation of $(H, a d)$ was defined to be a pair $\left(H_{t}, a_{t}\right)$ where $H_{t}$ is a $k[[t]]$-Hopf algebra given by $H_{t}=H \otimes k[[t]]$ with all Hopf algebra structures inherited by extending those on $H_{t}$. Let $\left.A=(H \otimes k[[t]]) /\left(t^{2}\right)\right)$ and the Hopf algebra structure maps $\mu, \Delta, \epsilon, \eta, S$ be inherited on $A$. As a vector space $A$ can be regarded as $H \oplus t H$

Recall that a solution to the YBE, $R$-matrix $R_{\text {ad }}$ is induced from the adjoint map. Then from the constructions of the adjoint cohomology from the point of view of the deformation theory, we obtain the following deformation of this $R$-matrix induced from the adjoint map.

Theorem 5. Let $\phi \in Z_{\text {ad }}^{2}(H ; H)$ be an adjoint 2-cocycle. Then the map $R: A \otimes A \rightarrow A \otimes A$ defined by $R=R_{\mathrm{ad}+t \phi}$ satisfies the $Y B E$.

Proof. The equalities of Lemma 2 hold in the quotient $A=(H \otimes k[[t]]) /\left(t^{2}\right)$, where $n=1$ and the modulus $t^{2}$ is considered. These cocycle conditions, on the other hand, were formulated from the motivation from Lemma 1 for the induced $R$-matrix $R_{\text {ad }}$ to satisfy the YBE. Hence these two lemmas imply the theorem.

Example. In Subsection 5.3, the adjoint map ad was computed for the bosonization $H$ of the superline, with basis $\{1, g, x, g x\}$, as well as a general 2-cocycle $\phi$ with three free variables $\alpha, \beta, \gamma$ written by

$$
\begin{aligned}
& \phi(g \otimes x)=\phi(g \otimes g x)=\alpha 1, \quad \phi(x \otimes g)=-\alpha 1, \quad \phi(g x \otimes g)=\gamma g \\
& \phi(g x \otimes x)=\beta 1+\gamma x, \quad \phi(g x \otimes g x)=-\beta 1+\gamma x
\end{aligned}
$$

and zero otherwise. Thus we obtain the deformed solution to the YBE $R=R_{\mathrm{ad}+t \phi}$ on $A$ with three variables $t \alpha, t \beta, t \gamma$ of degree one.

\section{Concluding remarks}

In [7] we concluded with A Compendium of Questions regarding our discoveries. Here we attempt to address some of these questions by providing relationships between this paper and [7], and offer further questions for our future consideration.

It was pointed out in [7] that there was a clear distinction between the Hopf algebra case and the cocommutative coalgebra case as to why self-adjoint maps satisfy the YBE. In [7] a cohomology theory was constructed for the coalgebra case. In this paper, many of the same ideas and techniques, in particular deformations and diagrams, were used to construct a cohomology theory in the Hopf algebra case, with applications to the YBE and quandle cohomology.

The aspects that unify these two theories are deformations and a systematic process we call "diagrammatic infiltration." So far, these techniques have only been successful in defining coboundaries up through dimension 3. This is a deficit of the diagrammatic approach, but diagrams give direct applications to other algebraic problems such as the YBE and quandle cohomology, and suggest further applications to knot theory. By taking the trace as in Turaev's [21], for example, a new deformed version of a given invariant is expected to be obtained.

Many questions remain: Can 3-cocycles be used for solving the tetrahedral equation? Can they be used for knotted surface invariants? Can the coboundary maps be expressed skein theoretically? How are the deformations of $R$-matrices related to deformations of underlying Hopf algebras? When a Hopf algebra contains a coalgebra, such as the universal enveloping algebra and its Lie algebra together with the ground field of degree-zero part, what is the relation between the two theories developed in this paper and in [7]? How these theories, other than the same diagrammatic techniques, can be uniformly formulated, and to higher dimensions? 


\section{Acknowledgements}

JSC (NSF Grant DMS \#0301095, \#0603926) and MS (NSF Grant DMS \#0301089, \#0603876) gratefully acknowledge the support of the NSF without which substantial portions of the work would not have been possible. The opinions expressed in this paper do not reflect the opinions of the National Science Foundation or the Federal Government. JSC, ME, and MS have benefited from several detailed presentations on deformation theory that have been given by Jörg Feldvoss. AC acknowledges useful and on-going conversations with John Baez.

\section{References}

[1] N. Andruskiewitsch and M. Graña. From racks to pointed Hopf algebras. Adv. Math. 178 (2003), $177-243$.

[2] J. C. Baez and A. S. Crans. Higher-Dimensional Algebra VI: Lie 2-Algebras. Theory Appl. Categ. 12 (2004), 492-538.

[3] J. C. Baez and L. Langford. 2-tangles. Lett. Math. Phys. 43 (1998), 187-197.

[4] E. Brieskorn. Automorphic sets and singularities. In "Proc. AMS-IMS-SIAM Joint Summer Research Conference in Mathematical Sciences on Artin's Braid Group held at the University of California, Santa Cruz, California, July 13-26, 1986. J. S. Birman and A. Libgober, Eds. AMS, Providence, RI, 1988.". Contemp. Math., 78 (1988), 45-115.

[5] J. S. Carter, M. Elhamdadi, and M. Saito Twisted Quandle homology theory and cocycle knot invariants Algebr. Geom. Topol. 2 (2002), 95-135.

[6] J. S. Carter, D. Jelsovsky, S. Kamada, L. Langford, and M. Saito. Quandle cohomology and statesum invariants of knotted curves and surfaces. Trans. Amer. Math. Soc. 355 (2003), 3947-3989.

[7] J. S. Carter, A. Crans, M. Elhamdadi, and S. Saito. Cohomology of Categorical Self-Distributivity. To appear in Journal of Homotopy Theory and Related Structures; preprint math. GT/0607417.

[8] A. S. Crans. Lie 2-algebras. Ph.D. Dissertation, 2004, UC Riverside. Preprint math.QA/0409602.

[9] R. Fenn and C. Rourke. Racks and links in codimension two. J. Knot Theory Ramifications, 1 (1992), 343-406.

[10] M. Gerstenharber and S. D. Schack. Bialgebra cohomology, deformations, and quantum groups. Proc. Nat. Acad. Sci. U.S.A. 87 (1990), 478-481.

[11] M. A. Hennings. On solutions to the braid equation identified by Woronowicz. Lett. Math. Phys. 27 (1993), 13-17.

[12] D. Joyce. A classifying invariant of knots, the knot quandle. J. Pure Appl. Algebra, 23 (1982) 37-65.

[13] G. Kuperberg. Involutory Hopf algebras and 3-manifold invariants. Int. J. Math. 2 (1991), 41-66.

[14] S. Majid. A Quantum Groups Primer. London Math. Soc. Lecture Note Ser. 292. Cambridge Univ. Press, Cambridge, 2002.

[15] S. Majid. Foundations of Quantum Group Theory. Cambridge Univ. Press, Cambridge, 1995.

[16] M. Markl and J. D. Stasheff. Deformation theory via deviations. J. Algebra, 170 (1994), 122-155.

[17] M. Markl and A. Voronov. PROPped up graph cohomology. To appear in Maninfest; preprint http://arxiv.org/pdf/math/0307081.

[18] S. Matveev. Distributive groupoids in knot theory. Mat. Sb. (N.S.) 119 (161) (1982), 78-88 (160).

[19] N. Reshetikhin and V. G. Turaev. Invariants of 3-manifolds via link polynomials and quantum groups. Invent. Math. 103 (1991), 547-597.

[20] J.-L. Tu. Groupoid cohomology and extensions. Trans. Amer. Math. Soc. 358 (2006), 4721-4747.

[21] V. G. Turaev. The Yang-Baxter equation and invariants of links. Invent. Math. 92 (1988), 527-553.

[22] S. L. Woronowicz. Solutions of the braid equation related to a Hopf algebra. Lett. Math. Phys. 23 (1991), 143-145. 